\title{
THE LIMITS OF APPLICABILITY OF THE LINEARIZATION METHOD IN CALCULATING SMALL-TIME REACHABLE SETS ${ }^{1}$
}

\author{
Mikhail I. Gusev \\ Krasovskii Institute of Mathematics and Mechanics, \\ Ural Branch of the Russian Academy of Sciences, \\ 16 S. Kovalevskaya Str., Ekaterinburg, 620990, Russia \\ gmi@imm.uran.ru
}

\begin{abstract}
The reachable sets of nonlinear systems are usually quite complicated. They, as a rule, are non-convex and arranged to have rather complex behavior. In this paper, the asymptotic behavior of reachable sets of nonlinear control-affine systems on small time intervals is studied. We assume that the initial state of the system is fixed, and the control is bounded in the $\mathbb{L}_{2}$-norm. The subject of the study is the applicability of the linearization method for a sufficiently small length of the time interval. We provide sufficient conditions under which the reachable set of a nonlinear system is convex and asymptotically equal to the reachable set of a linearized system. The concept of asymptotic equality is defined in terms of the Banach-Mazur metric in the space of sets. The conditions depend on the behavior of the controllability Gramian of the linearized system - the smallest eigenvalue of the Gramian should not tend to zero too quickly when the length of the time interval tends to zero. The indicated asymptotic behavior occurs for a reasonably wide class of secondorder nonlinear control systems but can be violated for systems of higher dimension. The results of numerical simulation illustrate the theoretical conclusions of the paper.
\end{abstract}

Key words: Nonlinear control systems, Small-time reachable sets, Asymptotics, Integral constraints, Linearization.

\section{Introduction}

The paper explores the properties of reachable sets of control-affine nonlinear systems with integral constraints over small time intervals. The geometric structure of reachable sets plays an important role in control theory, in particular, in solving problems of control synthesis. Smalltime reachable sets under pointwise (geometric) constraints on control were studied by C. Lobry, H. Sussmann, A. J. Krener, H. Schattler, and C. I. Byrnes (see, for example, [12, 19]). In general, the reachable sets of nonlinear systems are not convex and may have a quite complicated structure $[1,3,11,13-15,20,21]$. When some of the parameters of a control system are small (initial deviations from the equilibrium position, disturbances at the input of the system, etc.), the behavior of the system can often be judged by the action of its linear approximation. Here we find out under what conditions this linearization approach is applicable when constructing reachable sets on small time intervals. Will these sets be close to reachable sets of a linearized system? In this paper, we study reachable sets for control-affine systems on small time intervals with integral quadratic constraints on the controls. Reachable sets of nonlinear systems with integral constraints were studied in $[5-7,16]$. If a system is linear, its reachable set is an ellipsoid in the state space. Therefore, an ellipsoid is the reachable set of a linearized system. To establish the proximity of the reachable sets of original and linearized systems, it is necessary first to find out in which

\footnotetext{
${ }^{1}$ This work is supported by the Research and Education Center of IMM UB of RAS in the framework of the Ural Mathematical Center (project "Set-Valued Dynamics in Control and Estimation Problems for Dynamical Systems with Uncertainty").
} 
cases the reachable set of the original nonlinear system is convex. B. Polyak [17] proved that a nonlinear image of a small ball in a Hilbert space is convex under some regularity assumptions on the mapping. Using this result, he showed that reachable sets of a nonlinear control system are convex if constraints on the control are given by a ball of a sufficiently small radius in $\mathbb{L}_{2}$ and the linearized system is controllable [16]. Using a time change, we reduce the problem of constructing the reachable set of a system on a small time interval to a similar problem on a unit interval. With this replacement, the integral constraints are given by a ball of small radius, and we apply Theorem 1 from [17] to propose sufficient conditions for the convexity of small-time reachable sets. The application of these conditions requires a study of the asymptotic behavior of the controllability Gramian of the linearized system depending on a small parameter.

Another question is how to evaluate the degree of proximity of reachable sets for small lengths of time intervals. These sets contract to a single-point set as the interval length tends to zero, so the Hausdorff metric is not enough for this purpose. Here we use the concept of asymptotic equality of sets introduced in [4] and based on the Banach-Mazur metric.

The paper is arranged as follows. In Section 1, we introduce the concept of asymptotic equality of sets using the Banach-Mazur metric. We prove several auxiliary statements concerning the connection of this concept with the properties of support functions. In Section 2, we consider relations between the images of a Hilbert ball under nonlinear mapping depending on a small parameter and under its linear approximation. Further, we apply these results to the study of the asymptotic behavior of the reachable sets of nonlinear systems with integral control constraints. We formulate sufficient conditions for the asymptotic equality of reachable sets of nonlinear and linearized systems. These conditions depend on the asymptotic behavior of the controllability Gramian of the linearized system. The asymptotic behavior of the smallest eigenvalue of the controllability Gramian for a time-invariant linear control system with a single input is studied in Section 3. In Section 4, we apply the obtained asymptotics to the study of reachable sets for affine-control nonlinear systems on a small time interval. We give two examples of nonlinear two-dimensional systems and present the results of numerical simulations.

\section{Asymptotic equality of sets}

Let $X, Y \subset \mathbb{R}^{n}$ be convex compact sets. We assume that the zero vector is an interior point of each of these sets. The Banach-Mazur distance $\rho(X, Y)$ between $X$ and $Y$ is defined by the equality

$$
\rho(X, Y):=\log (r(X, Y) \cdot r(Y, X)), \quad r(X, Y)=\inf \{t \geq 1: t X \supset Y\} .
$$

For convex closed sets $X$ and $Y$, the inclusion $t X \supset Y$ holds if and only if

$$
t \delta(y \mid X) \geq \delta(y \mid Y), \quad \forall y \in \mathbb{R}^{n}, \quad\|y\|=1,
$$

where $\delta(y \mid X)$ is the support function of the set $X$ :

$$
\delta(y \mid X):=\sup \{(y, x): x \in X\}, \quad y \in \mathbb{R}^{n} .
$$

Hence, we have the formula

$$
r(X, Y)=\max \left\{1, \sup _{\|y\|=1} \frac{\delta(y \mid X)}{\delta(y \mid Y)}\right\}
$$

Note that, due to the condition $0 \in \operatorname{int} Y$, the inequality $\delta(y \mid Y)>0$ holds for $\|y\| \neq 0$.

Suppose further that the sets under consideration depend on a small positive parameter $\varepsilon$, $X=X(\varepsilon)$ and $Y=Y(\varepsilon)$ are convex compact sets, and the zero vector is an interior point of each 
of these sets for $0<\varepsilon \leq \varepsilon_{0}$. We also assume that the multivalued mappings $X(\varepsilon)$ and $Y(\varepsilon)$ are bounded. The sets $X(\varepsilon)$ and $Y(\varepsilon)$ are called asymptotically equal [4] if $\rho(X(\varepsilon), Y(\varepsilon)) \rightarrow 0$ as $\varepsilon \rightarrow 0$.

We use the notation

$$
\Delta_{X Y}(y, \varepsilon):=\frac{\delta(y \mid X(\varepsilon))}{\delta(y \mid Y(\varepsilon))}, \quad \Delta_{Y X}(y, \varepsilon):=\frac{\delta(y \mid Y(\varepsilon))}{\delta(y \mid X(\varepsilon))} .
$$

Formula (2.1) implies the following statement.

Lemma 1. In order to $\rho(X(\varepsilon), Y(\varepsilon)) \rightarrow 0$ as $\varepsilon \rightarrow 0$, it is necessary and sufficient that

$$
\lim _{\varepsilon \rightarrow 0} \Delta_{X Y}(y, \varepsilon)=1 \text { uniformly in } y, \quad\|y\|=1 .
$$

P r o o f. It follows from (2.2) that $\lim _{\varepsilon \rightarrow 0} \sup _{\|y\|=1} \Delta_{X Y}(y, \varepsilon)=1$. Since $\Delta_{X Y}(y, \varepsilon)$. $\Delta_{Y X}(y, \varepsilon)=1$, we have $\lim _{\varepsilon \rightarrow 0} \sup _{\|y\|=1} \Delta_{Y X}(y, \varepsilon)=1$. From formula (2.1), we find that $r(X(\varepsilon), Y(\varepsilon)) \rightarrow 1$ and $r(Y(\varepsilon), X(\varepsilon)) \rightarrow 1$; therefore, $\rho(X(\varepsilon), Y(\varepsilon)) \rightarrow 0$ as $\varepsilon \rightarrow 0$.

To prove the necessity of condition (2.2), suppose, on the contrary, that this condition is violated. Then there exist $1>\sigma>0$ and a sequence $\varepsilon_{k} \rightarrow 0$ such that the following relations are valid for an infinite number of the sequence terms:

$$
\sup _{\|y\|=1} \Delta_{X Y}\left(y, \varepsilon_{k}\right) \geq 1+\sigma \quad \text { or } \sup _{\|y\|=1} \Delta_{X Y}\left(y, \varepsilon_{k}\right) \leq 1-\sigma .
$$

In the former case, we have $r\left(X\left(\varepsilon_{k}\right), Y\left(\varepsilon_{k}\right)\right) \geq 1+\sigma$ and, therefore, $\rho\left(X\left(\varepsilon_{k}\right), Y\left(\varepsilon_{k}\right)\right) \geq \log (1+\sigma)>0$. In the latter case, we obtain

$$
\Delta_{X Y}\left(y, \varepsilon_{k}\right) \leq 1-\sigma, \quad \forall y, \quad\|y\|=1,
$$

and hence

$$
\sup _{\|y\|=1} \Delta_{Y X}\left(y, \varepsilon_{k}\right) \geq \frac{1}{1-\sigma}
$$

This implies that

$$
\rho\left(X\left(\varepsilon_{k}\right), Y\left(\varepsilon_{k}\right)\right) \geq \log \left(1+\frac{\sigma}{1-\sigma}\right)>0
$$

for an infinite number of the sequence terms $\varepsilon_{k}$. This contradicts the convergence of $\rho\left(X\left(\varepsilon_{k}\right), Y\left(\varepsilon_{k}\right)\right)$ to zero.

The condition $\rho(X(\varepsilon), Y(\varepsilon)) \rightarrow 0$ implies that $h(X(\varepsilon), Y(\varepsilon)) \rightarrow 0$ as $\varepsilon \rightarrow 0$, where $h$ denotes the Hausdorff distance between the sets. Indeed, by Lemma 1, relation (2.2) holds in this case. Therefore, for any $\sigma>0$, there exists $\bar{\varepsilon}$ such that the inequalities

$$
\Delta_{X Y}(y, \varepsilon) \leq 1+\sigma, \quad \Delta_{Y X}(y, \varepsilon) \leq 1+\sigma
$$

hold for all $y \in \mathbb{R}^{n},\|y\|=1,0<\varepsilon \leq \bar{\varepsilon}$. These inequalities imply the estimate

$$
h(X(\varepsilon), Y(\varepsilon))=\sup _{\|y\|=1}|\delta(y \mid X(\varepsilon))-\delta(y \mid Y(\varepsilon))| \leq \sigma \max \left\{\sup _{\|y\|=1} \delta(y \mid Y(\varepsilon)) \sup _{\|y\|=1} \delta(y \mid X(\varepsilon))\right\},
$$

which means that $h(X(\varepsilon), Y(\varepsilon)) \rightarrow 0$ as $\varepsilon \rightarrow 0$.

The converse is not true, as the following example shows. Let

$$
X(\varepsilon)=\left\{x \in \mathbb{R}^{2}:\left|x_{1}\right| \leq \varepsilon,\left|x_{2}\right| \leq \varepsilon\right\}, \quad Y(\varepsilon)=\left\{x \in \mathbb{R}^{2}: x_{1}^{2}+x_{2}^{2} \leq \varepsilon^{2}\right\} .
$$

Then $h(X(\varepsilon), Y(\varepsilon))=(\sqrt{2}-1) \varepsilon \rightarrow 0$ and $\rho(X(\varepsilon), Y(\varepsilon))=\log \sqrt{2}>0$. Nevertheless, under the additional assumption about the rate of convergence of the Hausdorff distance between the sets, we prove in Theorem 1 that $\rho(X(\varepsilon), Y(\varepsilon)) \rightarrow 0$ as $\varepsilon \rightarrow 0$.

For $A \subset \mathbb{R}^{n}$, define $\delta_{\min }(A):=\inf _{\|y\|=1} \delta(y \mid A)$. 
Theorem 1. The following conditions are sufficient for $\rho(X(\varepsilon), Y(\varepsilon)) \rightarrow 0$ as $\varepsilon \rightarrow 0$ :

$$
\lim _{\varepsilon \rightarrow 0} h(X(\varepsilon), Y(\varepsilon))=0, \quad \lim _{\varepsilon \rightarrow 0} \frac{h(X(\varepsilon), Y(\varepsilon))}{\delta_{\min }(Y(\varepsilon))}=0 .
$$

P r o o f. Let $h(\varepsilon)=h(X(\varepsilon), Y(\varepsilon))$ and $\delta(\varepsilon)=\delta_{\min }(Y(\varepsilon))$. From the equality

$$
h(\varepsilon)=h(X(\varepsilon), Y(\varepsilon))=\sup _{\|y\|=1}|\delta(y \mid X(\varepsilon))-\delta(y \mid Y(\varepsilon))|,
$$

it follows that

$$
-h(\varepsilon) \leq \delta(y \mid X(\varepsilon))-\delta(y \mid Y(\varepsilon)) \leq h(\varepsilon)
$$

for all $y \in \mathbb{R}^{n},\|y\|=1$. Dividing these inequalities by a positive value $\delta(y \mid Y(\varepsilon))$, we get

$$
\left|\sup _{\|y\|=1} \frac{\delta(y \mid X(\varepsilon))}{\delta(y \mid Y(\varepsilon))}-1\right| \leq \sup _{\|y\|=1} \frac{h(\varepsilon)}{\delta(y \mid Y(\varepsilon))} \leq \frac{h(\varepsilon)}{\delta(\varepsilon)} .
$$

Dividing these inequalities by $\delta(y \mid X(\varepsilon))$ and taking into account that, in view of the conditions of the theorem, $\delta_{\min }(\varepsilon)-h(\varepsilon)>0$ for sufficiently small $\varepsilon$, we get

$$
\left|\sup _{\|y\|=1} \frac{\delta(y \mid Y(\varepsilon))}{\delta(y \mid X(\varepsilon))}-1\right| \leq \sup _{\|y\|=1} \frac{h(\varepsilon)}{\delta(y \mid X(\varepsilon))} \leq \frac{h(\varepsilon)}{\delta(\varepsilon)-h(\varepsilon)} .
$$

From these inequalities, we obtain relations (2.2) and hence, by Lemma $1, \rho(X(\varepsilon), Y(\varepsilon)) \rightarrow 0$ as $\varepsilon \rightarrow 0$.

Note that the definition of $\rho(X, Y)$ is symmetrical with respect to the sets $X$ and $Y$. Therefore, in the statement of the theorem, $\delta_{\min }(Y(\varepsilon))$ can be replaced by $\delta_{\min }(X(\varepsilon))$.

\section{Small-time reachable sets of nonlinear systems}

\subsection{Auxiliary results}

Let $X$ and $Y$ be Banach spaces. Denote by $B_{X}\left(a, \mu_{0}\right) \subset X$ the ball of radius $\mu_{0}$ centered at $a$. Consider a mapping $F_{\varepsilon}: B_{X}\left(a, \mu_{0}\right) \rightarrow Y$ depending on a parameter $\varepsilon, 0<\varepsilon<\varepsilon_{0}$.

Assumption 1. The mapping $F_{\varepsilon}(x)$ has a Fréchet derivative with respect to $x$, which satisfies the Lipschitz condition on $B_{X}\left(a, \mu_{0}\right)$

$$
\left\|F_{\varepsilon}^{\prime}\left(x_{1}\right)-F_{\varepsilon}^{\prime}\left(x_{2}\right)\right\| \leq L(\varepsilon)\left\|x_{1}-x_{2}\right\|, \quad x_{1}, x_{2} \in B_{X}\left(a, \mu_{0}\right), \quad \varepsilon \in\left(0, \varepsilon_{0}\right],
$$

where $L(\varepsilon)$ is a function bounded on $\left(0, \varepsilon_{0}\right]$.

Let a function $\mu(\varepsilon) \operatorname{map}\left(0, \varepsilon_{0}\right]$ to $\left(0, \mu_{0}\right]$. Assume that $\mu(\varepsilon) \rightarrow 0$ as $\varepsilon \rightarrow 0$. Denote by $G_{\varepsilon}$ the image of the ball $B_{X}(a, \mu(\varepsilon))$ under the mapping $F_{\varepsilon}$ :

$$
G_{\varepsilon}:=\left\{F_{\varepsilon}(x): x \in B_{X}(a, \mu(\varepsilon))\right\}
$$

Theorem 2. Suppose that condition (3.1) holds. Then

$$
h\left(\left(\operatorname{co} G_{\varepsilon}-F_{\varepsilon}(a)\right), \mu(\varepsilon) F_{\varepsilon}^{\prime}(a) B_{X}(0,1)\right) \leq L(\varepsilon) \mu^{2}(\varepsilon),
$$

where $h$ is the Hausdorff distance between sets and co $G$ denotes the convex hull of the set $G$. 
P r o o f. The proof follows from the proof of Theorem 1 in [10].

Let $X$ and $Y$ be real Hilbert spaces. Suppose that a mapping $F: X \supset B_{X}\left(a, \mu_{0}\right) \rightarrow Y$ is differentiable and its Frechét derivative $F^{\prime}$ satisfies the Lipschitz condition with constant $L$. Let a mapping $F$ be regular at the point $a$, i.e., let the operator $F^{\prime}(a): X \rightarrow Y$ be a surjection. The latter property implies the existence of a positive number $\gamma$ such that $\left\|F^{\prime}(a)^{*} y\right\| \geq \gamma\|y\|$ for all $y \in Y$, which is equivalent to the inequality

$$
\left(F^{\prime}(a) F^{\prime}(a)^{*} y, y\right) \geq \nu\|y\|^{2}
$$

for all $y \in Y$, where $\nu=\gamma^{2}$ is the smallest eigenvalue of the self-adjoint operator $F^{\prime}(a) F^{\prime}(a)^{*}$. Here $(\cdot, \cdot)$ is the bilinear form for the duality between $Y$ and the space $Y^{*}$ conjugate to $Y, F^{\prime}(a)^{*}$ stands for the operator adjoint to a bounded linear operator $F^{\prime}(a)$. In [17, Theorem 1], it is shown that, if the inequality

$$
\mu \leq \min \left\{\mu_{0}, \frac{\sqrt{\nu}}{2 L}\right\}
$$

holds, then the image of the ball $B_{X}(a, \mu)$, i.e., the set $G=\left\{F(x): x \in B_{X}(a, \mu)\right\}$, is convex.

In what follows, we assume that $X$ is a Hilbert space and $Y=\mathbb{R}^{n}$ is a finite-dimensional Euclidean space. Consider the family of operators $F_{\varepsilon}$ assuming that each mapping $F_{\varepsilon}$ is regular at the point $a$. Denote by $\nu(\varepsilon)$ the smallest eigenvalue of the operator (matrix)

$$
W_{\varepsilon}:=F_{\varepsilon}^{\prime}(a) F_{\varepsilon}^{\prime}(a)^{*}
$$

Note that in this case the set $E_{\varepsilon}:=F_{\varepsilon}^{\prime}(a) B_{X}(0,1)$ is a finite-dimensional ellipsoid defined by the relation

$$
E_{\varepsilon}=\left\{x \in \mathbb{R}^{n}: x^{\top} W_{\varepsilon}^{-1} x \leq 1\right\},
$$

and $\sqrt{\nu(\varepsilon)}$ is the length of its smallest semiaxis. Theorem 2 implies the following statement.

Corollary 1. Suppose that $\mu(\varepsilon) \leq \sqrt{\nu(\varepsilon)} /(2 L(\varepsilon))$. Then the set $G_{\varepsilon}$ is convex and

$$
h\left(G_{\varepsilon}, F_{\varepsilon}(a)+\mu(\varepsilon) E_{\varepsilon}\right) \leq L(\varepsilon) \mu^{2}(\varepsilon) .
$$

P r o o f. The convexity of $G_{\varepsilon}$ follows from inequality (3.2). Hence, under the conditions of the corollary, $G_{\varepsilon}=\operatorname{co} G_{\varepsilon}$. Using Theorem 2, we get

$$
h\left(G_{\varepsilon}, F_{\varepsilon}(a)+\mu(\varepsilon) E_{\varepsilon}\right)=h\left(\left(\operatorname{co} G_{\varepsilon}-F_{\varepsilon}(a)\right), \mu(\varepsilon) F_{\varepsilon}^{\prime}(a) B_{X}(0,1)\right) \leq L(\varepsilon) \mu^{2}(\varepsilon) .
$$

Corollary 2. Suppose that $\mu(\varepsilon) L(\varepsilon) / \sqrt{\nu(\varepsilon)} \rightarrow 0$ as $\varepsilon \rightarrow 0$. Then the set $G_{\varepsilon}$ is convex for sufficiently small $\varepsilon$ and

$$
\rho\left(G_{\varepsilon}-F_{\varepsilon}(a), \mu(\varepsilon) E_{\varepsilon}\right) \rightarrow 0 \text { as } \varepsilon \rightarrow 0 .
$$

P r o o f. Since $\mu(\varepsilon) L(\varepsilon) / \sqrt{\nu(\varepsilon)} \rightarrow 0$, we have $\mu(\varepsilon) L(\varepsilon) / \sqrt{\nu(\varepsilon)} \leq 1 / 2$ for all sufficiently small $\varepsilon$. For these $\varepsilon$, we have $\mu(\varepsilon) \leq \sqrt{\nu(\varepsilon)} /(2 L(\varepsilon))$, hence, $G_{\varepsilon}$ is convex. Consider two convex compact sets depending on $\varepsilon$ :

$$
X(\varepsilon)=G_{\varepsilon}-F_{\varepsilon}(a), \quad Y(\varepsilon)=\mu(\varepsilon) E_{\varepsilon} .
$$

Calculating the value $\delta(\varepsilon)=\delta_{\min }(Y(\varepsilon)$ ) (see Theorem 1), we get

$$
\delta(y \mid Y(\varepsilon))=\mu(\varepsilon) \sqrt{y^{\top} W_{\varepsilon} y}, \quad \delta(\varepsilon)=\min _{\|y\|=1} \delta(y \mid Y(\varepsilon))=\mu(\varepsilon) \sqrt{\nu(\varepsilon)} .
$$


Since $\delta(\varepsilon)>0$ for $\varepsilon>0$, we have $0 \in \operatorname{int} Y(\varepsilon)$. It follows from Lyusternik's theorem [2] that $F_{\varepsilon}(a) \in \operatorname{int} G_{\varepsilon}$ for $\varepsilon>0$. Thus, the zero vector is an interior point of $X(\varepsilon)$ and $Y(\varepsilon)$ for all sufficiently small positive $\varepsilon$.

In view of the inequality $h(X(\varepsilon), Y(\varepsilon)) \leq L(\varepsilon) \mu(\varepsilon)^{2}$, we have

$$
\frac{h(X(\varepsilon), Y(\varepsilon))}{\delta(\varepsilon)} \leq \frac{L(\varepsilon) \mu(\varepsilon)^{2}}{\mu(\varepsilon) \sqrt{\nu(\varepsilon)}}=\frac{\mu(\varepsilon) L(\varepsilon)}{\sqrt{\nu(\varepsilon)}} \rightarrow 0 \text { as } \varepsilon \rightarrow 0 .
$$

By Theorem 1, we find that $\rho(X(\varepsilon), Y(\varepsilon)) \rightarrow 0$.

Thereby, the set $G_{\varepsilon}-F_{\varepsilon}(a)$ is asymptotically equal to $\mu(\varepsilon) E_{\varepsilon}$. This means that the image of the ball $B(a, \mu(\varepsilon))$ under the nonlinear transformation $F_{\varepsilon}$ is close in shape to the ellipsoid $F_{\varepsilon}(a)+\mu(\varepsilon) E_{\varepsilon}$. The latter is the result of transforming the ball by means of a linear approximation of $F_{\varepsilon}$ at the point $a$.

\subsection{Small-time reachable sets}

Consider a nonlinear control-affine system

$$
\dot{x}(t)=f_{1}(x(t))+f_{2}(x(t)) u(t), \quad 0 \leq t \leq \varepsilon \leq \bar{\varepsilon}, \quad x(0)=x^{0},
$$

where $x \in \mathbb{R}^{n}$ and $u \in \mathbb{R}^{r}$ are state and control inputs, respectively, and $\bar{\varepsilon}>0$. The initial state $x^{0}$ is assumed to be fixed. Denote by $\mathbb{L}_{2}[0, \bar{\varepsilon}]$ the Hilbert space of square integrable functions $[0, \bar{\varepsilon}] \rightarrow \mathbb{R}^{r}$. Constraints on controls are given in the form

$$
u(\cdot) \in B(0, \mu),
$$

where $B(0, \mu):=\left\{u(\cdot) \in \mathbb{L}_{2}[0, \bar{\varepsilon}]:(u(\cdot), u(\cdot)) \leq \mu^{2}\right\}$ is a ball of radius $\mu>0$ centered at zero and

$$
(u(\cdot), u(\cdot))=\| u(\cdot)) \|_{\mathbb{L}_{2}[0, \bar{\varepsilon}]}^{2}=\int_{0}^{\bar{\varepsilon}} u^{\top}(t) u(t) d t .
$$

Suppose that, for any $u(\cdot) \in B(0, \mu)$, there exists a unique solution $x(t, u(\cdot))$ of system $(3.3)$, this solution is defined on $[0, \bar{\varepsilon}]$, and all trajectories starting from $x^{0}$ and corresponding to the controls from the ball $B(0, \mu)$ belong to a compact set $D$. Assume also that the functions $f_{1}$ and $f_{2}$ have Lipschitz continuous derivatives on $D$.

Let $G(\varepsilon, \mu)$ be the reachable set of system (3.3) at time $\varepsilon \in[0, \bar{\varepsilon}]$ under integral constraints

$$
G(\varepsilon, \mu):=\left\{x \in \mathbb{R}^{n}: \exists u(\cdot) \in B(0, \mu), x=x(\varepsilon, u(\cdot))\right\} .
$$

Since $\|u(\cdot)\|_{\mathbb{L}_{2}[0, \varepsilon]} \leq\|u(\cdot)\|_{\mathbb{L}_{2}[0, \bar{\varepsilon}]}$, the set $G(\varepsilon, \mu)$ can be written as follows:

$$
G(\varepsilon, \mu)=\left\{x \in \mathbb{R}^{n}: \exists u(\cdot),\|u(\cdot)\|_{\mathbb{L}_{2}[0, \varepsilon]} \leq \mu, x=x(\varepsilon, u(\cdot))\right\} .
$$

We study the behavior of reachable sets $G(\varepsilon, \mu)$ under the assumption that $\varepsilon$ is a small number. Using a time change, we reduce the problem of describing reachable sets on the time interval $[0, \varepsilon]$ to a similar problem on the interval $[0,1]$ for another system whose equations and integral constraints on the control depend on $\varepsilon$.

Representing $t$ in the form $t=\varepsilon \tau$, we set $y(\tau)=x(\varepsilon \tau)$ and $v(\tau)=\varepsilon u(\varepsilon \tau)$. Then

$$
\dot{y}(\tau)=\varepsilon f_{1}(y(\tau))+f_{2}(y(\tau)) v(\tau), \quad 0 \leq \tau \leq 1, \quad y(0)=x^{0},
$$


with the following constraint on the new control $v(\cdot)$ :

$$
\int_{0}^{1} v^{\top}(t) v(t) d t \leq(\mu \sqrt{\varepsilon})^{2} .
$$

The trajectories of system (3.4), (3.5) belong to the compact set $D$ if $\varepsilon \leq \bar{\varepsilon}$.

Define $\mu(\varepsilon):=\mu \sqrt{\varepsilon}$. Let $\tilde{G}(1, \mu)$ be the reachable set of system (3.4):

$$
\tilde{G}(1, \mu):=\left\{y \in \mathbb{R}^{n}: \exists v(\cdot) \in B(0, \mu) \subset \mathbb{L}_{2}[0,1], y=y(1, v(\cdot))\right\} .
$$

Hereinafter, we use the same notation $B(0, \mu)$ for balls in the spaces $\mathbb{L}_{2}[0, b]$ with different $b$. Besides, for simplicity, we omit the time interval in the notation of the space $\mathbb{L}_{2}$ if this does not cause misunderstanding.

Define a family of mappings $F_{\varepsilon}: \mathbb{L}_{2}[0,1] \rightarrow \mathbb{R}^{n}$ by the equality $F_{\varepsilon}(v(\cdot))=y_{\varepsilon}(1, v(\cdot))$, where $y_{\varepsilon}(t, v(\cdot))$ is the solution of system $(3.4)$ corresponding to $v(\cdot)$. Since $y(1, v(\cdot))=x\left(t_{1}, u(\cdot)\right)$, we have the equality

$$
\tilde{G}(1, \mu(\varepsilon))=G(\varepsilon, \mu)=\left\{F_{\varepsilon}(v(\cdot)): v(\cdot) \in B(0, \mu(\varepsilon))\right\} .
$$

The mapping $F_{\varepsilon}(v(\cdot))$ is differentiable; its derivative is defined as follows [10]:

$$
F_{\varepsilon}^{\prime}(v(\cdot)) \Delta v(\cdot)=\Delta y(1, \Delta v(\cdot)),
$$

where $\Delta y(\tau)$ is the solution of system (3.4) linearized along the trajectory $(y(\tau, v(\cdot)), v(\cdot))$

$$
\dot{\Delta} y(\tau)=\varepsilon A(\tau) \Delta y(\tau)+B(\tau) \Delta v(\tau), \quad \tau \in[0,1], \quad \Delta y(0)=0 .
$$

Here

$$
A(\tau)=\frac{\partial f_{1}}{\partial x}(y(\tau))+\sum_{i=1}^{r} \frac{\partial f_{2}^{i}}{\partial x}(y(\tau)) v_{i}(\tau), \quad B(\tau)=f_{2}(y(\tau)) .
$$

The following statement is true.

Proposition 1. [8,9] The mapping $F_{\varepsilon}^{\prime}(v(\cdot))$ is Lipschitz continuous on $B(0, \mu(\varepsilon))$ with the constant $L(\varepsilon)=L_{0}+L_{1} \varepsilon\left(L_{0}, L_{1} \geq 0\right)$. If all elements of the matrix $f_{2}$ in the equation of the system are independent of the state (i.e., $f_{2}(x)=f_{2}$ is a constant matrix), then $L_{0}=0$.

We can use Corollary 2 proved above to describe the reachable sets on small time intervals. In this case, $a=0 \in \mathbb{L}_{2}$ is the zero control and the derivative $F_{\varepsilon}^{\prime}(0)$ is defined by equation (3.6) corresponding to system (3.4) linearized along the trajectory $(y(\tau, 0), 0)$. Here $y(\tau, 0)$ is a solution of (3.4) with $v(\tau) \equiv 0, \tau \in[0,1]$. In this case,

$$
A(\tau)=\frac{\partial f_{1}}{\partial x}(y(\tau)), \quad B(\tau)=f_{2}(y(\tau)) .
$$

Consider a linear control system

$$
\dot{z}(\tau)=\varepsilon A(\tau) z(\tau)+B(\tau) u(\tau), \quad \tau \in[0,1],
$$

with continuous matrices $A(\tau)$ and $B(\tau)$.

Definition 1. The symmetric matrix $W_{\varepsilon}(\tau)$ defined by the equality

$$
W_{\varepsilon}(\tau)=\int_{0}^{\tau} X_{\varepsilon}(\tau, s) B(s) B^{\top}(s) X_{\varepsilon}^{\top}(\tau, s) d s,
$$

where $X_{\varepsilon}(\tau, s)$ is the fundamental Cauchy matrix of system $(3.7)\left(\dot{X}_{\varepsilon}(\tau, s)=\varepsilon A(\tau) X_{\varepsilon}(\tau, s)\right.$, $X(s, s)=I)$ is called the controllability Gramian of the control system (3.7). 
Differentiating equality (3.8), it is easy to see that $W_{\varepsilon}(t)$ is a solution of the linear differential equation

$$
\dot{W}_{\varepsilon}(\tau)=\varepsilon A(\tau) W_{\varepsilon}(\tau)+\varepsilon W_{\varepsilon}(\tau) A^{\top}(\tau)+B(\tau) B^{\top}(\tau), \quad W_{\varepsilon}(0)=0 .
$$

The system is completely controllable on the interval $[0,1]$ if and only if $W_{\varepsilon}(1)$ is positive definite. It is known (see, for example, $[14,16]$ ) that, in this case, the reachable set under the constraint

$$
\int_{0}^{1} u^{\top}(\tau) u(\tau) d \tau \leq \mu^{2}
$$

is an ellipsoid defined as the set of solutions of the inequality $x^{\top} W_{\varepsilon}^{-1}(1) x \leq \mu^{2}$.

From the above, we can conclude that the matrix $W_{\varepsilon}=F_{\varepsilon}^{\prime}(0) F_{\varepsilon}^{\prime}(0)^{*}$ coincides with the controllability Gramian $W_{\varepsilon}(1)$ of system (3.6) and the ellipsoid $\mu(\varepsilon) E_{\varepsilon}=\hat{G}(\varepsilon, \mu)$ is the reachable set at time 1 of system (3.6) under constraint (3.5). Note that $F_{\varepsilon}(0)$ equals to $x(\varepsilon, 0)$. Taking into account that $\mu(\varepsilon)=\mu \sqrt{\varepsilon}$, we arrive at the following statement.

Theorem 3. Let $\nu(\varepsilon)$ be the smallest eigenvalue of the controllability Gramian $W_{\varepsilon}^{-1}(1)$ of the linearized system (3.6). Suppose that $L(\varepsilon) \sqrt{\varepsilon} / \sqrt{\nu(\varepsilon)} \rightarrow 0$ as $\varepsilon \rightarrow 0$. Then the reachable set $G(\varepsilon, \mu)$ is convex for sufficiently small $\varepsilon$ and

$$
\rho(G(\varepsilon, \mu)-x(\varepsilon, 0), \hat{G}(\varepsilon, \mu)) \rightarrow 0 \text { as } \varepsilon \rightarrow 0,
$$

where $\hat{G}(\varepsilon, \mu)$ is the reachable set of the linearized system (3.6).

Using the reverse time change, it is easy to show that $\hat{G}(\varepsilon, \mu))$ is the reachable set at time $\varepsilon$ for the linearized system (3.3). Thus, Theorem 3 states that, under proper asymptotic behavior of the smallest eigenvalue of the controllability Gramian, the small-time reachable set is asymptotically equal to the reachable set of the linearized system. The asymptotic behavior of the Gramian for the case of linear autonomous systems is studied in the next section.

\section{Time-invariant systems on a small time interval}

\subsection{Asymptotics of the smallest eigenvalue of the controllability Gramian}

Consider a linear time-invariant control system

$$
\dot{x}(t)=\varepsilon A x(t)+B u(t), \quad t \in[0,1],
$$

where $x \in \mathbb{R}^{n}, u \in \mathbb{R}^{r}$, and $\varepsilon>0$ is a small parameter. If the pair $(A, B)$ is completely controllable, then $(\varepsilon A, B)$ is also controllable for all $\varepsilon \neq 0$. In this case, the smallest eigenvalue of the controllability Gramian $\nu(\varepsilon)=\nu\left(W_{\varepsilon}(1)\right)$ is positive for all $\varepsilon>0$. In this section, we study the asymptotic behavior of $\nu(\varepsilon)$ for small $\varepsilon$.

Consider the controllability Gramian $W_{\varepsilon}(t)$ of system (4.1). The matrix $W_{\varepsilon}(t), t>0$, is positive definite for every $\varepsilon \neq 0$ if and only if the pair $(A, B)$ is completely controllable. Let us look for $W_{\varepsilon}(t)$ as the sum of series in powers of $\varepsilon$ :

$$
W_{\varepsilon}(t)=V_{0}(t)+\varepsilon V_{1}(t)+\varepsilon^{2} V_{2}(t)+\cdots, \quad V_{k}(0)=0, \quad k=0,1, \ldots .
$$

Differentiating (4.2) and equating coefficients at equal powers of $\varepsilon$, we get

$$
\dot{V}_{0}(t)=B B^{\top}, \quad \dot{V}_{k}(t)=A V_{k-1}(t)+V_{k-1}(t) A^{\top}, \quad k=1,2, \ldots .
$$


Integrating equations (4.3), we get

$$
V_{0}(t)=t U_{0}, \quad V_{i}(t)=\frac{t^{i+1}}{(i+1) !} A U_{i}, \quad i=1,2, \ldots,
$$

where

$$
U_{0}=B B^{\top}, \quad U_{i}=A U_{i-1}+U_{i-1} A^{\top}, \quad k=1,2, \ldots .
$$

Thus, for $W_{\varepsilon}=W_{\varepsilon}(1)$, we have

$$
W_{\varepsilon}=\sum_{k=0}^{\infty} \frac{\varepsilon^{k}}{(k+1) !} U_{k} .
$$

In view of the estimate $\left\|U_{k}\right\| \leq 2\|A\|\left\|U_{k-1}\right\| \leq 2^{k}\|A\|^{k}\left\|U_{0}\right\|$, series (4.4) and (4.2) are majorized by the converging series

$$
\sum_{k=0}^{\infty} \frac{(2 \varepsilon\|A\|)^{k}}{(k+1) !}\left\|U_{0}\right\| .
$$

Here $\|A\|$ is the spectral matrix norm induced by the Euclidean vector norm. As a result, we find that the matrix $W_{\varepsilon}=W_{\varepsilon}(1)$ is represented as the sum of series (4.4) uniformly convergent on every bounded subset of $\mathbb{R}$.

Note that all matrices $U_{k}$ in (4.4) are symmetric but not necessarily positive semi-definite. For $U_{0}$, we obviously have $\nu\left(U_{0}\right) \geq 0$. If $\nu\left(U_{0}\right)>0$, then there exists $\alpha>0$ such that $\nu\left(W_{\varepsilon}\right) \geq \alpha$ for sufficiently small $\varepsilon$. Further, we assume that $\nu\left(U_{0}\right)=0$, hence $\nu\left(W_{\varepsilon}\right) \rightarrow 0$ as $\varepsilon \rightarrow 0$.

Definition 2. [18] A pair $(A, B)$ is linearly equivalent to a pair $\left(A_{1}, B_{1}\right)$ if there exists a nonsingular matrix $S$ such that $A_{1}=S A S^{-1}$ and $B_{1}=S B$.

Linear equivalent pairs generate equations of the same control system in different coordinate systems. A pair $(A, B)$ is controllable iff $\left(A_{1}, B_{1}\right)$ is controllable. The asymptotic behavior of the controllability Gramians of linear equivalent pairs is the same.

Proposition 2. $\left[9\right.$, Lemma 1] Let $(A, B)$ and $\left(A_{1}, B_{1}\right)$ be linearly equivalent pairs, and let $W_{\varepsilon}$ and $W_{\varepsilon}^{1}$ be the corresponding controllability Gramians. There exist $\alpha>0$ and $\beta>0$ such that

$$
\alpha \nu\left(W_{\varepsilon}\right) \leq \nu\left(W_{\varepsilon}^{1}\right) \leq \beta \nu\left(W_{\varepsilon}\right)
$$

for all $\varepsilon$.

Consider systems with single control. In this case, $A$ is an $n \times n$ matrix and $B$ is an $n$-dimensional column-vector.

Theorem 4. [9, Theorem 1] Assume that a system is completely controllable. If $n=2$, then there exist $\alpha>0$ and $\beta>0$ such that the following inequality holds for all sufficiently small $\varepsilon>0$ :

$$
\alpha \varepsilon^{2} \leq \nu\left(W_{\varepsilon}\right) \leq \beta \varepsilon^{2} .
$$

If $n \geq 3$, then there exists $\beta>0$ such that

$$
0<\nu\left(W_{\varepsilon}\right) \leq \beta \varepsilon^{2 n-2}
$$

for all sufficiently small $\varepsilon>0$.

The proof of this theorem is based on reducing the control system to the Frobenius form. 


\subsection{Small-time reachable sets of time-invariant systems}

Consider an autonomous control system with a single input

$$
\dot{x}(t)=f(x(t))+B u(t), \quad x(0)=x^{0}, \quad 0 \leq t \leq \varepsilon,
$$

where $x \in \mathbb{R}^{n}, u \in \mathbb{R}, f: \mathbb{R}^{n} \rightarrow \mathbb{R}^{n}$ is a continuously differential mapping, $B$ is an $n \times 1$ matrix (a column-vector), and $x^{0}$ is a fixed initial state, with control variables subjected to the quadratic integral constraints

$$
\int_{0}^{\varepsilon} u^{2}(t) d t \leq \mu^{2}
$$

Suppose, as above, that there exists a compact set $D \subset \mathbb{R}^{n}$ containing all trajectories of system (4.5) and that $f(x)$ has a Lipschitz continuous derivative on this set.

Denote by $A(t)=\frac{\partial f}{\partial x}(x(t, 0))$ the matrix of the system linearized along the trajectory $x(t, 0)$ corresponding to the zero control. Suppose that $f\left(x^{0}\right)=0$. In this case, $x(t, 0) \equiv 0$ and, hence,

$$
A(t)=\frac{\partial f}{\partial x}(x(t, 0))=\frac{\partial f}{\partial x}\left(x^{0}\right)=A
$$

is a constant matrix. Let $W_{\varepsilon}$ be the controllability Gramian of the pair $(\varepsilon A, B)$ on the interval $[0,1]$, and let $\nu(\varepsilon)$ be the smallest eigenvalue of $W_{\varepsilon}$. If the pair $(A, B)$ is controllable, then, by Theorem $4, \nu(\varepsilon) \geq \alpha \varepsilon^{2}$ if $n=2$ and $\nu\left(W_{\varepsilon}\right) \leq \beta \varepsilon^{4}$ if $n \geq 3$ for some $\alpha, \beta>0$.

From Theorem 3 we obtain the following statement.

Corollary 3. Let $n=2$, and let system (4.5) linearized at the point $x^{0}$ be completely controllable. Then the reachable set $G(\varepsilon, \mu)$ is convex for all sufficiently small $\varepsilon$ and asymptotically equal to the reachable set of the linearized system.

P r o o f. In the conditions of the theorem, we have $L(\varepsilon)=L_{1} \varepsilon$ (see Proposition 1) and $\nu(\varepsilon) \geq \alpha \varepsilon^{2}$. This implies that $L(\varepsilon) \sqrt{\varepsilon} / \sqrt{\nu(\varepsilon)} \leq\left(L_{1} / \sqrt{\alpha}\right) \sqrt{\varepsilon} \rightarrow 0$ as $\varepsilon \rightarrow 0$.

Note than the sufficient conditions for the convexity of $G(\varepsilon)$ are not satisfied for a system with a single input for $n \geq 3$.

\subsection{Examples}

As an illustrative example, consider the Duffing oscillator

$$
\dot{x}_{1}=x_{2}, \quad \dot{x}_{2}=-x_{1}-10 x_{1}^{3}+u, \quad 0 \leq t \leq \varepsilon
$$

which describes the motion of a nonlinear stiff spring on impact of an external force $u$, with integral constraints

$$
\int_{0}^{\varepsilon} u^{2}(t) d t \leq \mu^{2}
$$

and zero initial state $x_{1}(0)=0, x_{2}(0)=0$.

Consider the Lyapunov-type function

$$
V(x)=V\left(x_{1}, x_{2}\right)=\frac{5}{2} x_{1}^{4}+\frac{1}{2} x_{1}^{2}+\frac{1}{2} x_{2}^{2} .
$$

Differentiating $V(x(t))$ along an arbitrary trajectory of the system and applying an analog of Grownwall's Lemma [23], we find that all trajectories of system (4.6) belong to the compact set $D=\left\{x \in \mathbb{R}^{2}: V(x) \leq \mu^{2} \varepsilon\right\}$ (see [22]). 

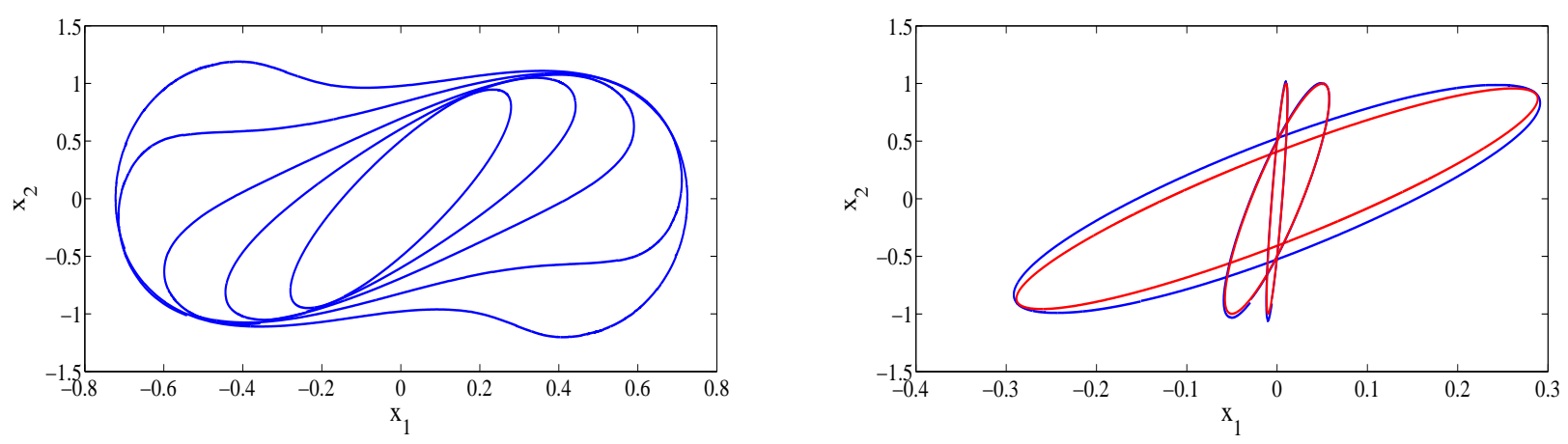

Figure 1. Reachable sets of Duffing oscillator

System (4.6) linearized along $x(t) \equiv 0$ after a time change

$$
\dot{x}_{1}=\varepsilon x_{2}, \quad \dot{x}_{2}=-x_{1}+u, \quad x(0)=(0,0), \quad 0 \leq \tau \leq 1,
$$

is completely controllable. From Corollary 3 it follows that, for small $\varepsilon$, the reachable sets $G(\varepsilon)$ in this example are convex sets close in shape to ellipsoids.

The results of the numerical simulation are shown in the figure that follows. These results are obtained with the use of an algorithm based on Pontryagin's maximum principle for boundary trajectories.

Fig. 1 shows the results of numerical simulation for this example. Its left-hand side exhibits the plot of the boundaries of the reachable set at times $\varepsilon=0.5,0.7,0.9,1.2$, and 1.5 , respectively. A larger set in the figure corresponds to a larger value of $\varepsilon$. This plot indicates that the reachable sets for smaller values of $\varepsilon$ are convex and look like ellipsoids. The right-hand side of the figure corresponds to smaller $\varepsilon$. Here the boundaries of reachable sets of the nonlinear system are shown in blue and of the linearized system in red. Note that the reachable sets contract to zero as $\varepsilon \rightarrow 0$. In order to make the picture more informative, we multiply each of the sets by a scaling factor $s(\varepsilon)$ depending on $\varepsilon$. The resulting ellipsoids tend to a degenerate ellipsoid (vertical segment) as $\varepsilon \rightarrow 0$.

As another example, consider a bilinear system

$$
\left\{\begin{array}{l}
\dot{x}_{1}=x_{2} u_{1}-x_{1} u_{2} \\
\dot{x}_{2}=-x_{1} u_{1}-x_{2} u_{2}
\end{array}\right.
$$

with initial state given by the equalities $x_{1}(0)=1$ and $x_{2}(0)=0$. It is known that, under control constraints in the form

$$
\left|u_{1}(t)\right| \leq 1, \quad\left|u_{2}(t)\right| \leq 1, \quad 0 \leq t \leq \varepsilon,
$$

the reachable set $G(\varepsilon)$ is non-convex for any $\varepsilon>0$ [18]. Consider further the integral constraints on the control

$$
\int_{0}^{\varepsilon}\left(u_{1}^{2}(t)+u_{2}^{2}(t)\right) d t \leq 1
$$

All trajectories of the system belong to a compact set on the plane. This fact could be easily proved by using the transition to the polar coordinates. The matrices $A$ and $B$ of the system linearized along the trajectory $x(t) \equiv(1,0)$ have the following form:

$$
A=\left(\begin{array}{ll}
0 & 0 \\
0 & 0
\end{array}\right), \quad B=\left(\begin{array}{cc}
0 & -1 \\
-1 & 0
\end{array}\right) .
$$


The system is completely controllable and the controllability Gramian $W_{\varepsilon}$ is independent of $\varepsilon$ :

$$
W_{\varepsilon}=B B^{\top}=\left(\begin{array}{ll}
1 & 0 \\
0 & 1
\end{array}\right) .
$$

Since $\nu\left(W_{\varepsilon}\right)=\nu(\varepsilon)$ and the Lipschitz constant $L(\varepsilon)$ is independent of $\varepsilon$, we have

$$
L(\varepsilon) \sqrt{\varepsilon} / \sqrt{\nu(\varepsilon)} \rightarrow 0
$$

as $\varepsilon \rightarrow 0$. Consequently, the reachable sets $G(\varepsilon)$ are convex for sufficiently small $\varepsilon$ and asymptotically equal to ellipsoids (see also [10]).

\section{REFERENCES}

1. Baier R., Gerdts M., Xausa I. Approximation of reachable sets using optimal control algorithms. Numer. Algebra Control Optim., 2013. Vol. 3, No. 3. P. 519-548. DOI: 10.3934/naco.2013.3.519

2. Dmitruk A.V., Milyutin A.A., Osmolovskii N.P. Lyusternik's theorem and the theory of extrema. Russian Math. Surveys, 1980. Vol. 35, No. 6. P. 11-51. DOI: 10.1070/RM1980v035n06ABEH001973

3. Filippova T.F. Ellipsoidal estimates of reachable sets for control systems with nonlinear terms. IFACPapersOnLine, 2017. Vol. 50, No. 1. P. 15355-15360. DOI: 10.1016/j.ifacol.2017.08.2460

4. Goncharova E., Ovseevich A. Small-time reachable sets of linear systems with integral control constraints: birth of the shape of a reachable set. J. Optim. Theory Appl., 2016. Vol. 168. P. 615-624. DOI: $10.1007 / \mathrm{s} 10957-015-0754-4$

5. Guseinov K.G., Ozer O., Akyar E., Ushakov V.N. The approximation of reachable sets of control systems with integral constraint on controls. Nonlinear Differ. Equ. Appl., 2007. Vol. 14. P. 57-73. DOI: $10.1007 / \mathrm{s} 00030-006-4036-6$

6. Guseinov Kh. G., Nazlipinar A. S. Attainable sets of the control system with limited resources. Trudy Inst. Mat. i Mekh. UrO RAN, 2010. Vol. 16, No. 5. P. 261-268.

7. Gusev M. On reachability analysis of nonlinear systems with joint integral constraints. In: Lecture Notes in Comput. Sci., vol. 10665: Large-Scale Scientific Computing. LSSC 2017. Lirkov I., Margenov S. (eds.) Cham: Springer, 2018. P. 219-227. DOI: 10.1007/978-3-319-73441-523

8. Gusev M.I., Zykov I.V. On extremal properties of the boundary points of reachable sets for control systems with integral constraints. Proc. Steklov Inst. Math., 2018. Vol. 300. Suppl. 1. P. 114-125. DOI: $10.1134 /$ S0081543818020116

9. Gusev M. I. Estimates of the minimal eigenvalue of the controllability Gramian for a system containing a small parameter. In: Lecture Notes in Comput. Sci., vol. 11548: Int. Conf. Mathematical Optimization Theory and Operations Research. MOTOR 2019. Khachay M., Kochetov Y., Pardalos P. (eds.) Cham: Springer, 2019. P. 461-473. DOI: 10.1007/978-3-030-22629-9_32

10. Gusev M. I., Osipov I. O. Asimptoticheskoye povedeniye mnozhestv dostizhimosti na malykh vremennykh promezhutkakh [Asymptotic behavior of reachable sets on small time intervals]. Trudy Inst. Mat. Mekh. UrO RAN, 2019. Vol. 25, No. 3. P. 86-99. DOI: 10.21538/0134-4889-2019-25-3-86-99 (in Russian)

11. Kostousova E. K. On polyhedral estimates for reachable sets of discrete-time systems with bilinear uncertainty. Autom. Remote Control, 2011. Vol. 72. P. 1841-1851. DOI: 10.1134/S0005117911090062

12. Krener A. J., Schättler H. The structure of small-time reachable sets in low dimensions. SIAM J. Control Optim., 1989. Vol. 27. No. 1. P. 120-147. DOI: 10.1137/0327008

13. Kurzhanski A. B., Varaiya P. Dynamic optimization for reachability problems. J. Optim. Theory Appl., 2001. Vol. 108(2). P. 227-251. DOI: 10.1023/A:1026497115405

14. Kurzhanski A.B., Varaiya P. Dynamics and Control of Trajectory Tubes. Theory and Computation. Systems Control Found. Appl., vol. 85. Basel: Birkhäuser, 2014. 445 p. DOI: 10.1007/978-3-319-10277-1

15. Patsko V.S., Pyatko S. G., Fedotov A. A. Three-dimensional reachability set for a nonlinear control system. J. Comput. Syst. Sci. Int., 2003. Vol. 42. No. 3. P. 320-328.

16. Polyak B. T. Convexity of the reachable set of nonlinear systems under $L_{2}$ bounded controls. Dyn. Contin. Discrete Impuls. Syst. Ser. A Math. Anal., 2004. Vol. 11. P. 255-267.

17. Polyak B. T. Local programming. Comput. Math. Math. Phys., 2001. Vol. 41, No. 9. P. 1259-1266. 
18. Lee E. B. and Marcus L. Foundations of Optimal Control Theory. New York: J. Willey and Sons Inc., 1967. $576 \mathrm{p}$.

19. Schättler H. Small-time reachable sets and time-optimal feedback control. In: Nonsmooth Analysis and Geometric Methods in Deterministic Optimal Control. Vol. 78: The IMA Volumes in Mathematics and its Applications. Mordukhovich B.S., Sussmann H.J. (eds.) New York: Springer, 1996. P. 203-225. DOI: $10.1007 / 978-1-4613-8489-2 \_9$

20. Tochilin P. A. On the construction of nonconvex approximations to reach sets of piecewise linear systems. Differ. Equ., 2015. Vol. 51, No. 11. P. 1499-1511. DOI: 10.1134/S0012266115110117

21. Vdovin S. A., Taras'yev A. M., Ushakov V. N. Construction of the attainability set of a Brockett integrator. J. Appl. Math. Mech., 2004. Vol. 68, No. 5. P. 631-646. DOI: 10.1016/j.jappmathmech.2004.09.001

22. Zykov I. V. On external estimates of reachable sets of control systems with integral constraints. Izv. IMI UdGU, 2019. Vol. 53. P. 61-72. DOI: 10.20537/2226-3594-2019-53-06 (in Russian)

23. Walter W. Differential and Integral Inequalities. Berlin, Heidelberg: Springer-Verlag, 1970.354 p. DOI: $10.1007 / 978-3-642-86405-6$ 